\title{
Combining spatial components and Hilbert transforms to interpret ground-time-domain electromagnetic data
}

\author{
Jacques K. Desmarais ${ }^{1}$ and Richard S. Smith ${ }^{2}$
}

\begin{abstract}
We have developed a method for displaying or imaging data from a ground-time-domain electromagnetic system and for extracting the geometric parameters of a small conductor. The parameters are determined directly from the data using combinations of the spatial components of the secondary fields and their Hilbert transforms. The position of the target coincides with the peaks of the energy envelope (EE) or the $T$-component of the response. Here, the EE is the square root of the sum of the squares of the three spatial components and their Hilbert transforms, whereas the $T$-component response is an analogous quantity that excludes the Hilbert transform terms. Studies on synthetic models indicate that the $T$-component response is
\end{abstract}

sharper than the EE in most possible target orientations. Once the position of a body has been determined using the peak of the $T$-component response, the dip of the target can be quantified using the ratio of the full-width at half-magnitude (FWHM) of the $T$-component response and the $T$-component Hilbert transform response, which is analogous to the EE but excludes the untransformed quantities. Finally, once all other geometric parameters have been determined, the depth of the target can be evaluated using the FWHM of the $T$-component response. The proposed modeling method was tested over an anomaly acquired at the Coulon field site during an InfiniTEM survey in the Abitibi greenstone belt of Quebec. The extracted geometric parameters were consistent with the available geologic information.

\section{INTRODUCTION}

Geophysical practitioners are concerned with determining physical properties of the Earth through measurements of physical fields. For the purposes of interpreting potential fields, authors have advocated the use of the Hilbert transform within the context of the selfpotential method (Debeglia and Corpel, 1997; Akgün, 2001) and the magnetic method (Nabighian and Macnae, 1972; Nabighian 1974; Nabighian 1984; Bournas and Baker, 2001). The Hilbert transform is a relation between the real and imaginary parts of a complex function known as the analytic signal. The real part of the analytic signal is the original data, and the imaginary part is the Hilbert transform of the original data. The Hilbert transform follows directly from the properties of analytical functions (namely, the Cauchy-Riemann conditions and the Cauchy integral theorem) applied to the Fourier transform (Oppenheim et al., 1998). For geophysical applications, the Hilbert transform enables the determination of the complex trace of a real space-domain field.
By extending geophysical fields to the complex plane, one can build additional sets of equations that can be used to determine the physical parameters of the Earth (Akgün, 2001). As is shown by Nabighian and Macnae $(1972,1974)$, the absolute value of the analytic signal for magnetic fields associated with $2 \mathrm{D}$ models is independent of the orientation of the Earth's magnetic field. Nabighian (1984) extends the analysis to 3D.

Macnae (1984) shows that the absolute instantaneous secondary magnetic fields produced from conductors excited by a fixed transmitter and measured with a roving receiver are potential fields in the quasistatic approximation. Thus, measurements obtained from these systems may be considered as potential fields. Considering the success in interpreting potential-field data using the Hilbert transform, an extension to the case of ground time-domain electromagnetic (TDEM) data seems natural. In this spirit, Smith and Keating (1996) suggest interpreting electromagnetic data using an empirical quantity calculated in a similar manner to the absolute value of the

\footnotetext{
Manuscript received by the Editor 10 November 2014; revised manuscript received 17 March 2015; published online 19 June 2015.

${ }^{1}$ Presently University of Saskatchewan, Earth Sciences, Saskatoon, Saskatchewan, Canada; formerly Laurentian University, Sudbury, Ontario, Canada. E-mail: jkd788@mail.usask.ca.

${ }^{2}$ Laurentian University, Earth Sciences, Sudbury, Ontario, Canada. E-mail: rssmith@laurentian.ca.

(C) 2015 Society of Exploration Geophysicists. All rights reserved.
} 
analytic signal. They form a quantity known as the energy envelope (EE). The EE is calculated as follows (Smith and Keating, 1996):

$$
\mathrm{EE}=\sqrt{H_{x}^{2}+\tilde{H}_{x}^{2}+H_{y}^{2}+\tilde{H}_{y}^{2}+H_{z}^{2}+\tilde{H}_{z}^{2}},
$$

where $H$ denotes a magnetic field measurement, the subscript denotes the spatial component, and the tilde is the space-to-wavenumber domain Hilbert-transform operator. The space-to-wavenumber domain Hilbert transform of a $q$ th component measurement is expressed as (Oppenheim et al., 1998)

$$
\tilde{H}_{q}\left(k_{x}, k_{y}\right)=\frac{1}{\pi^{2}} \int_{-\infty}^{\infty} \frac{1}{k_{x}-r_{x}} d r_{x} \int_{-\infty}^{\infty} \frac{H_{q}\left(r_{x}, r_{y}\right)}{k_{y}-r_{y}} d r_{y},
$$

where $r_{x}, r_{y}$ are spatial variables and $k_{x}, k_{y}$ are the associated wavenumbers. The EE is a useful quantity because it yields a single peak over a vertical conductor when calculated on the data acquired with an airborne EM system. The EE also shows some asymmetry for fixed-wing systems. This asymmetry is a consequence of the manner in which the asymmetric transmitter receiver system couples to the conductor. Normalizing the individual components by the EE can remove some of this asymmetry.

Mercer (2012) proposes using the EE for generating maps of EM data acquired using the InfiniTEM system. The InfiniTEM system is a ground, dual-loop TDEM system. The two transmitter loops have reverse polarity and are connected in series in a figureeight-shaped loop design (Bérubé et al., 2006; Malo-Lalande, 2007; Brakni, 2011). The InfiniTEM system is beneficial because it generates a strong horizontal primary field between the two loops, which is useful for exploration in Archean terranes in which vertical conductors are abundant (Desmarais and Smith, 2015a). For the case of ground TDEM systems, the EE is analogous to the absolute value of the analytic signal. In the study of Mercer (2012), the EE generates a single peak anomaly over an anomalous body. For the case of a vertical conductor striking perpendicular to the traverse line (magnetic dipole moment parallel to the $[1,0,0]$ direction), Mercer (2012) finds that the EE is sharper than the $T$-component response. The $T$-component (or total) response is calculated as follows:

$$
H_{T}=\sqrt{H_{x}^{2}+H_{y}^{2}+H_{z}^{2}}
$$

In this study, we show that the $T$-component response is sharper than the EE in most of the possible target orientations. The spatial components of the secondary fields as well as their Hilbert transform are combined to determine the geometric parameters of the target.

Plotting quantities derived directly from the data, such as the $T$-component response or the $\mathrm{EE}$, are preferred over inversion schemes (such as Cox et al., 2010; Yang et al., 2014) because they are quicker to implement and do not require multiple runs with careful adjustment of the damping and regularization parameters. Moreover, we believe that the method presented herein provides a quicker alternative to conventional forward modeling, especially for targets at an offset and not striking perpendicular to the traverse line.

\section{METHODS}

The synthetic models investigated in this study are generated using the sphere model of Smith and Lee (2001). These authors derive the analytical expressions for the ideal impulse response of a sphere in free space excited by a dipolar field that is assumed to be locally uniform at the sphere. An ideal impulse response is defined as the magnetic-field response of the target once it has been subjected to an impulse in current in the transmitter (Smith and Lee, 2001). In our implementation, the sphere model is generalized to a more complex waveform by convolving the ideal impulse response with the InfiniTEM current waveform.

The primary field of the InfiniTEM system is computed using the method of Desmarais and Smith (2015a), who apply the sphere model of Smith and Lee (2001) to the InfiniTEM system. The primary field from the $800-\times 800$-m InfiniTEM loops was approximated as the field of 4624 infinitesimal magnetic dipoles. For loops of different sizes, the amount of dipoles are scaled proportionately. Modeling the field of a current-carrying loop of wire as a surface integral of the fields from magnetic dipoles was chosen for consistency with the model of Smith and Lee (2001).

The discrete conductor model of Smith and Lee (2001) can be adapted to compute the response of a sphere in which the induced currents in the target are constrained to flow in a specified direction. Mathematically, this process equates to projecting the magnetic dipole moment $\mathbf{m}_{\mathbf{s p}}$ of the sphere onto the normal $\mathbf{n}$ to the current flow path:

$$
\mathbf{m}_{\text {plate }}=\frac{\left(\mathbf{m}_{\mathrm{sp}} \cdot \mathbf{n}\right) \mathbf{n}}{n},
$$

where $\mathbf{m}_{\text {plate }}$ is the magnetic dipole moment of the plane containing the current flow path. As discussed in Desmarais and Smith (2015a) and Smith and Lee (2001), using the sphere model to approximate the response of a platelike conductor will generate the correct response shape and relative amplitude. However, the absolute response amplitude of the sphere may not equate to the absolute response amplitude of the equivalent plate model. This is not problematic for our study because the geometric parameters of anomalous targets can be estimated by implementing a normalization procedure. Ground TDEM data are normalized to the largest value of the $x$-, $y$-, and $z$-components, so that the response shape and relative amplitudes are preserved. As such, the sphere model is advantageous because it is less computationally demanding than an analogous plate model.

In the dipole approximation, the size and conductance of the target only affect the absolute amplitude of the responses (Desmarais and Smith, 2015b). Because any information on the absolute amplitude is lost during the normalization procedure, the fact that a sphere is different in shape than a plate is irrelevant. All that is important are the response shapes and relative amplitudes. As outlined by Desmarais and Smith (2015b), the response shapes and relative amplitudes of a spherical conductor are completely determined from the position of the body and the orientation of the plane containing the induced currents. Because equation 4 allows one to preserve these properties, a sphere may be considered as an ideal approximation to a plate for our purposes. Examples comparing the sphere model with that of an analogous plate model may be found in Desmarais and Smith (2015a) or Smith and Lee (2001). 
To generate the synthetic models, the InfiniTEM system is simulated with two 600- $\times 1000-\mathrm{m}$ loops along the $x$ - and $y$-directions, respectively. The loops are separated by a distance of $600 \mathrm{~m}$, and the traverse line spacing is $200 \mathrm{~m}$. The InfiniTEM waveform consists of an on-time ramp function containing an exponential turn on and a linear shutoff of a few $\mu \mathrm{s}$. The base frequency of the waveform was $30 \mathrm{~Hz}$, the duty cycle was $50 \%$, and the electric current in the transmitter was $24 \mathrm{~A}$. The target was a 50-m radius sphere with $10 \mathrm{~S} / \mathrm{m}$ conductivity.

Figure 1 shows an example of an impulse response function computed for a sphere whereby the induced currents are constrained to flow in a plane at a strike of $90^{\circ}$ and a dip of $90^{\circ}$. The strike and dip convention is outlined in Figure 2. The dip is measured in degrees below the traverse line, and the strike is measured from the positive $x$-direction. The sphere is located at a position of $[x, y, z]=$ $[144,200,100 \mathrm{~m}]$ with respect to the center of the configuration. The secondary magnetic field was calculated at the inductive limit $(t \rightarrow 0)$. As can be seen, the $x$-, $y$-, and $z$-component response shapes consist of many peaks and troughs, which are difficult to interpret. The response shape can be simplified using the EE as defined in equation 1 .

To calculate the Hilbert transform contained in the EE, it is necessary to first perform a fast Fourier transform (FFT) of the input data vector $\mathbf{H}$ with length $n$. The FFT is computed from the space domain to the wavenumber domain. The resulting Fourier domain signal $\overline{\mathbf{H}}$ is subsequently converted to a causal sequence. This can be achieved by applying a quadrature phase shift operator $\mathbf{h}$, whose $m$ th elements $\mathrm{h}_{m}$ take on the following values (Nabighian and Macnae, 1972):

$$
\mathrm{h}_{m}= \begin{cases}1, & m=n / 2+1 \\ 2, & m=2,3 \ldots, n / 2, \\ 0, & m=(n / 2)+2, \ldots, n\end{cases}
$$

Finally the first $n$ elements of the inverse FFT of $\mathbf{h} \overline{\mathbf{H}}$ yields the Hilbert transform vector $\tilde{\mathbf{H}}$ (Nabighian and Macnae, 1972).
If the data are plotted as profiles, a 1D Hilbert transform is calculated. If the data are plotted as plan maps, a 2D Hilbert transform is calculated.

For the purposes of studying its properties, we decompose the EE into two parts:

$$
\mathrm{EE}^{2}=H_{T}^{2}+\tilde{H}_{T}^{2}
$$

where $H_{T}$ is the $T$-component response as defined in equation 3 and $\tilde{H}_{T}$ the $T$-component Hilbert transform response:

$$
\tilde{H}_{T}=\sqrt{\tilde{H}_{x}^{2}+\tilde{H}_{y}^{2}+\tilde{H}_{z}^{2}} .
$$

To highlight the distinction between $H_{T}$ and $\tilde{H}_{T}$, henceforth we will refer to $H_{T}$ as the $T$-component response.

\section{RESULTS}

Figure 1d-1f shows plots of $H_{T}, \tilde{H}_{T}$, and $E E$ for the same synthetic model associated with Figure 1a-1c. Plan maps are generated using the values calculated along the survey lines, and values in between are determined through linear interpolation. Using this interpolation scheme, our method is currently only applicable to situations in which the body is located under a traverse line. Generalization to all possible target positions would require a more sophisticated interpolation scheme. As can be seen, the shapes of the responses with respect to the position of the target are simplified compared with those in Figure 1a-1c. All three quantities in Figure 3 generate a large peak near the anomalous body, so that they all provide viable options for plotting as plan maps or contours and for locating the body. The response shapes in Figure 1d-1f are simplified from those of Figure $1 \mathrm{a}-1 \mathrm{c}$, because $H_{T}, \tilde{H}_{T}$, and EE remove some of the asymmetry associated with coupling of the target with a complex transmitter-receiver system geometry.

We now study the manner in which $H_{T}, \tilde{H}_{T}$, and EE vary as a function of strike and dip. This can be accomplished by realizing a)

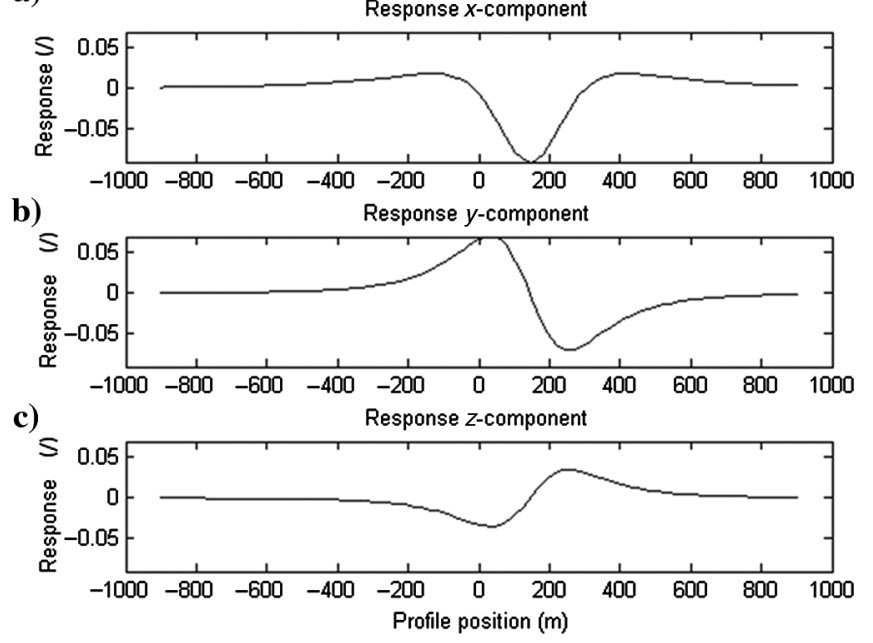

d)
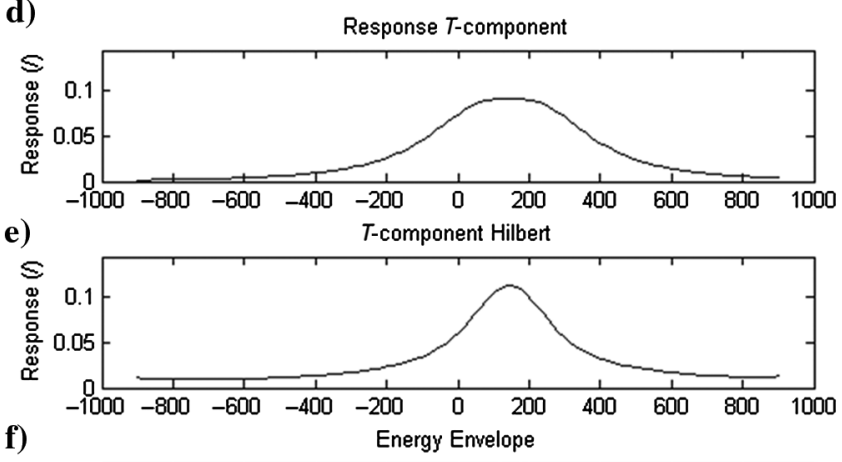

)

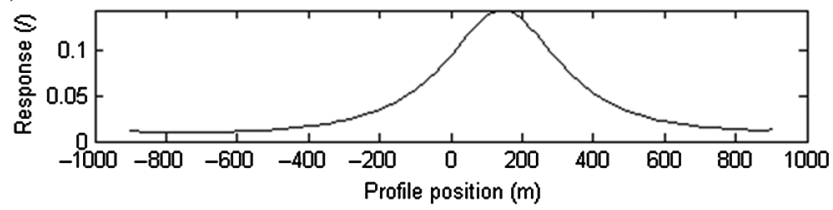

Figure 1. The three-component TDEM response of a sphere at a position of $[144,200,100 \mathrm{~m}]$ with respect to the center of the configuration. The traverse line along which the response has been calculated runs across the center of the configuration. The induced currents at the target are constrained to flow in a plane at a strike of $90^{\circ}$ and a dip of $90^{\circ}$ (dipole moment parallel to $[0,0,1]$ ). The inductive limit response is normalized to the largest value of the $x$-, $y$-, and $z$-components over the survey area, hence the units on the $y$ axis of this as well as subsequent curves are arbitrary. 
that the magnetic-field response of a platelike conductor in any arbitrary orientation can be expressed as a linear combination of the fields produced from target magnetic dipole moments oriented parallel to the $[1,0,0],[0,1,0]$, and $[0,0,1]$ directions. Figure 3 shows plan views of $H_{T}, \tilde{H}_{T}$, and $\mathrm{EE}$ for the case in which the induced currents at the target are constrained to flow in a plane at a strike of $90^{\circ}$ and a dip of $90^{\circ}$ (magnetic dipole moment parallel to $[1,0,0]$ ). The white dot indicates the location at which the computed quantities are at maximum. The gray dot indicates the position of the body. The black lines indicate the position of the loops, and the gray dashed lines indicate the position of the traverse lines. Figure 4 shows transverse and plan views of $H_{T}, \tilde{H}_{T}$, and $\mathrm{EE}$ for the case in which the induced currents at the target are constrained to flow in a plane at strike of $0^{\circ}$ and a dip of $90^{\circ}$ (magnetic dipole moment parallel to $[0,1,0]$ ). Finally, Figure 5 shows similar plots for the case in which the induced currents at the target are constrained to flow in a plane at a strike of $0^{\circ}$ and a dip of $0^{\circ}$ (magnetic dipole moment parallel to $[0,0,1])$.

In all three cases, the peaks of $\mathrm{EE}$ and $H_{T}$, coincide with the position of the target. The peak of $\tilde{H}_{T}$ is offset from the target when the magnetic dipole moment is parallel to $[0,1,0]$ and $[0,0,1]$, so that $\tilde{H}_{T}$ is more complicated to interpret than the other two quantities.

When the magnetic dipole moment is parallel to $[1,0,0]$ (Figure 3), The EE is sharper than the $T$-component response. However, when the magnetic dipole moment is parallel to $[0,1,0]$ or $[0,0,1]$, the $T$-component response is sharpest. Hence, the $T$-component response is best suited for data interpretation in most of the possible target orientations.

Once the position of the conductor has been determined from the location of the peak, other geometric parameters of the target can also be determined from the shape of the $T$-component response. To demonstrate how the parameters can be determined, we restrict our study to the case in which the target strikes perpendicular and is located below the traverse line. Conductors are often at small offsets to the traverse line because the TDEM responses are dominated by material close to the receiver. The requirement can normally be met to a fair approximation by selecting the traverse line with the largest response. Stratiform conductors (volcanogenic massive sulfide lenses, Mississippi-Valley-type, or epithermal deposits, etc.) can often be placed at a strike of $90^{\circ}$ by ensuring that the traverse line is perpendicular to the inferred strike of the bedrock.

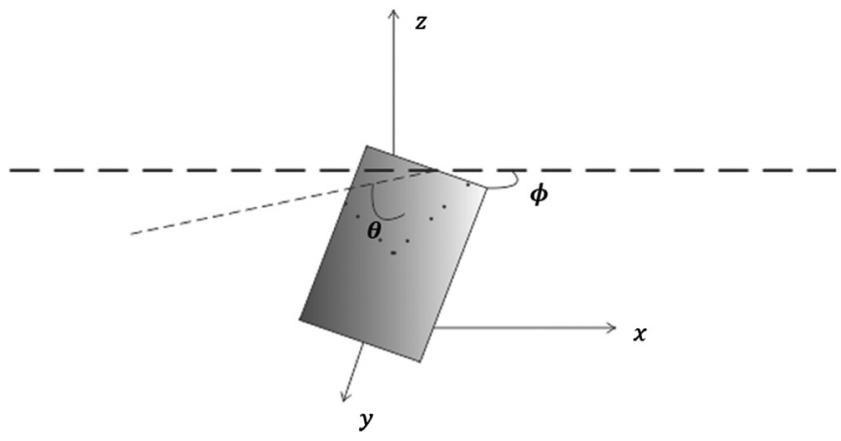

Figure 2. Schematic diagram showing the convention for the strike $(\phi)$ and the $\operatorname{dip}(\theta)$ of the plane containing the induced currents. The dip is measured from the horizontal, and the strike is measured from the positive $x$-direction.
In principle, the depth and dip could be determined from the properties of the $x$-, $y$-, and $z$-components; however, experience shows that the $x$-, $y$-, and $z$-components can dramatically change

a)

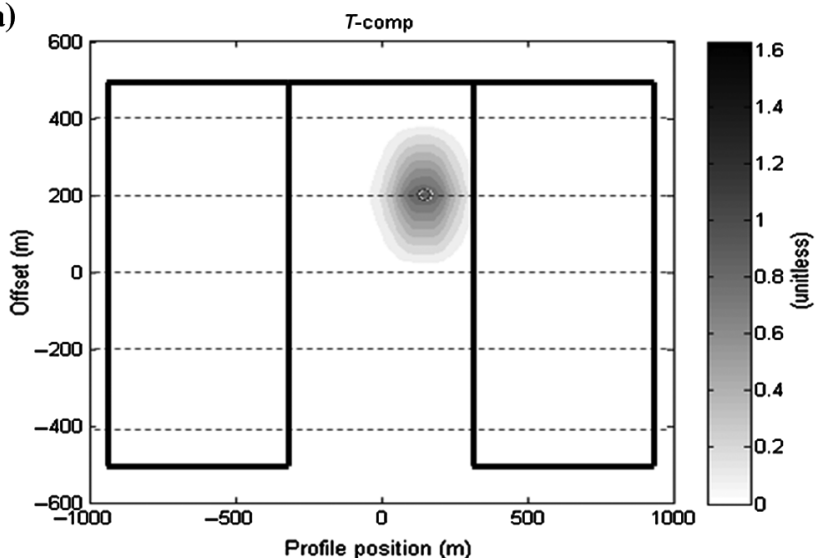

b)

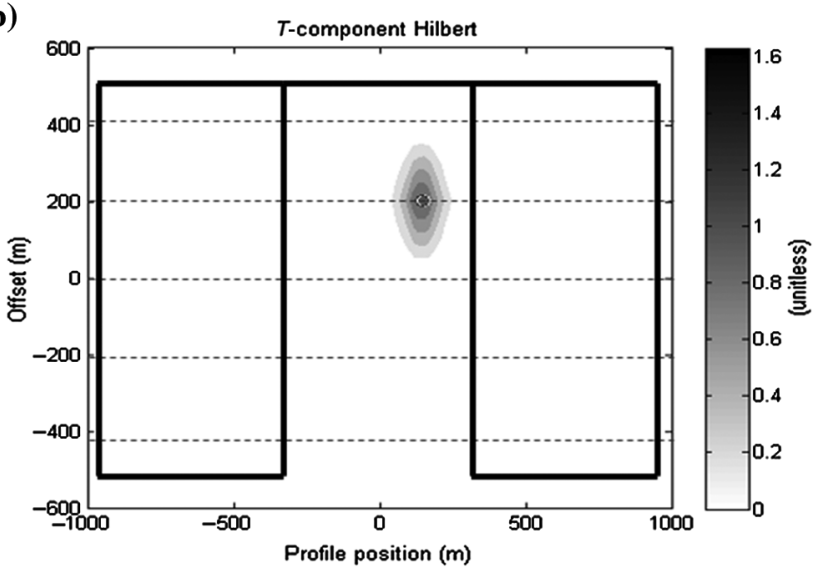

c)

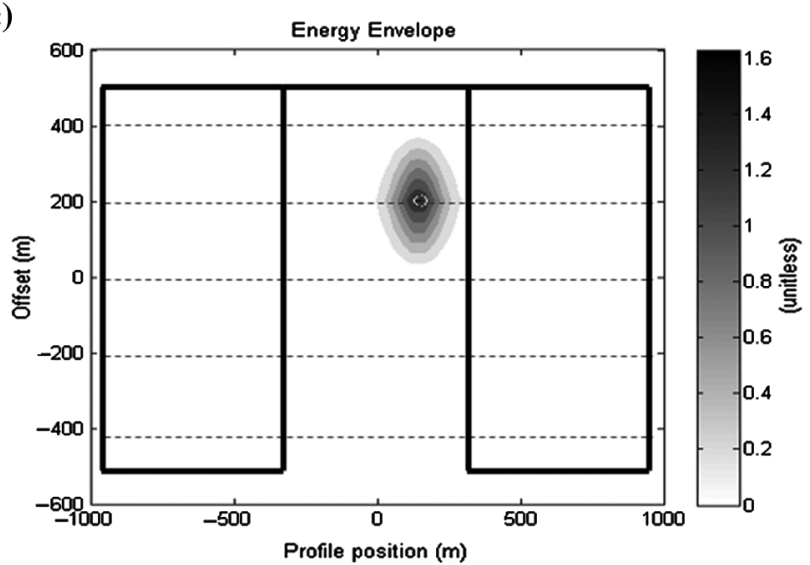

Figure 3. Here we have (a) $H_{T}$, (b) $\tilde{H}_{T}$, and (c) EE for the case in which the magnetic dipole moment is parallel to $[1,0,0]$. The white dot denotes the position at which the fields are at maximum. The gray dot denotes the position of the body. The black lines denote the position of the loops, and the gray dashed lines show the position of the traverse lines. In all three cases, the gray dot nearly coincides with the white dot so that the peaks of $H_{T}, \tilde{H}_{T}$, and EE coincide with the position of the target. The very small offset between the gray and white dots occurs because the position of the sphere, and the secondary fields are discretized on different grids. In this case, $\tilde{H}_{T}$ is the sharpest of all quantities, followed by EE. 
a)

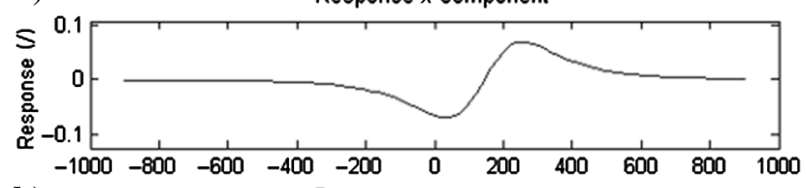

b)

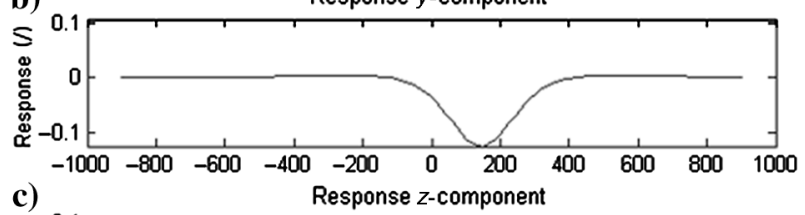

c)

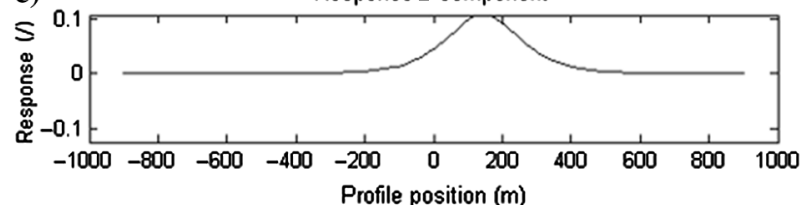

d)

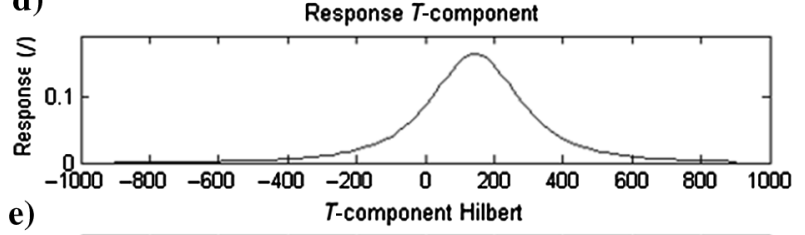

e)

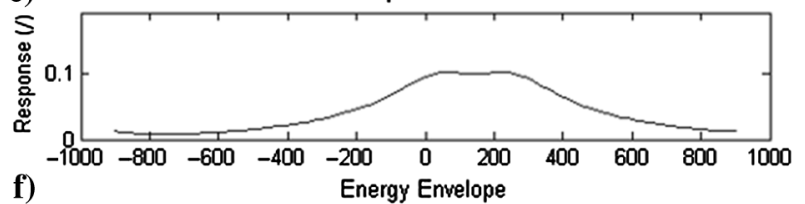

f)

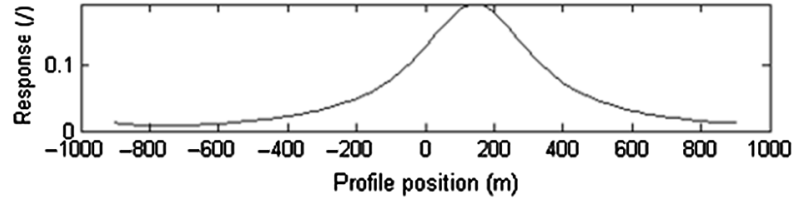

g)

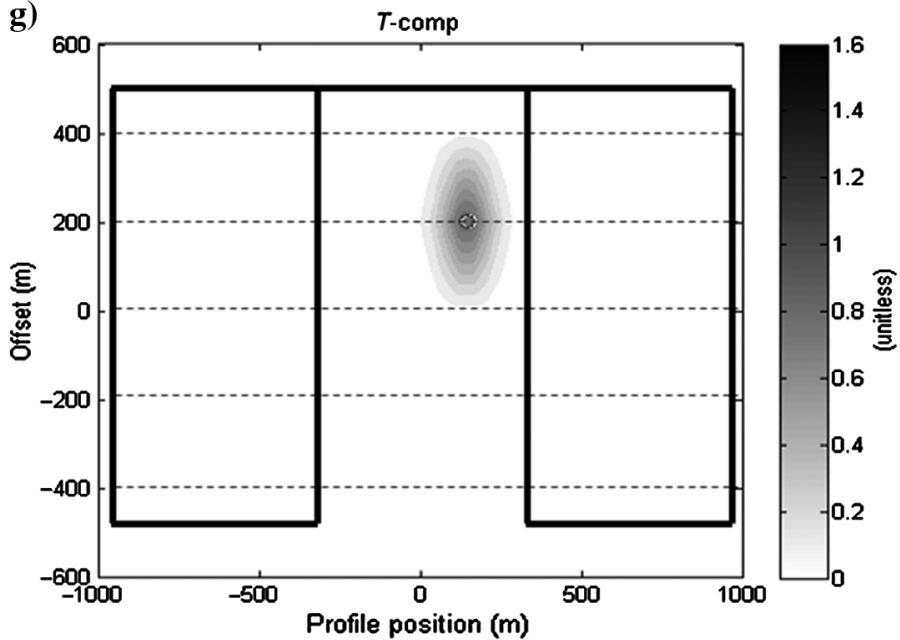

h)

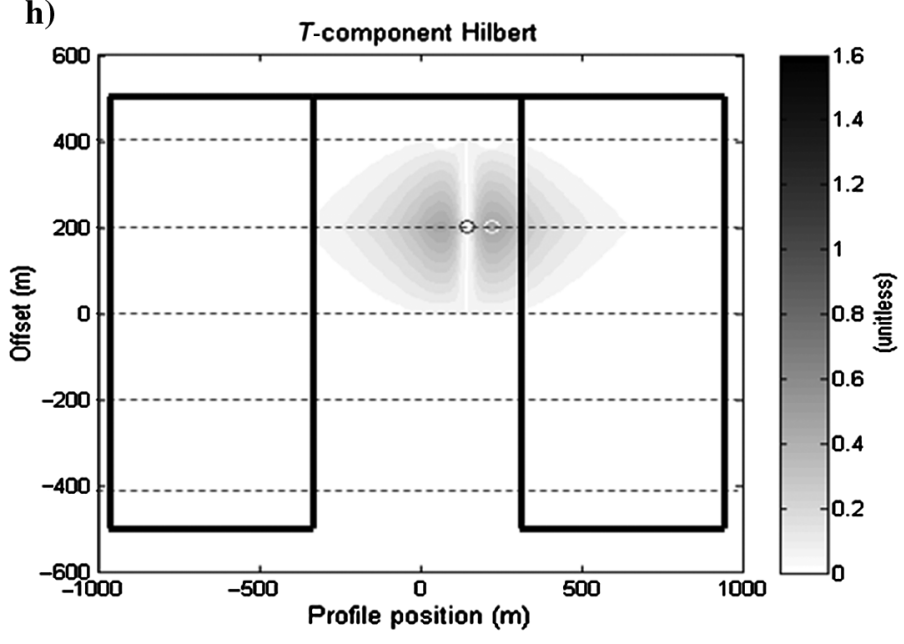

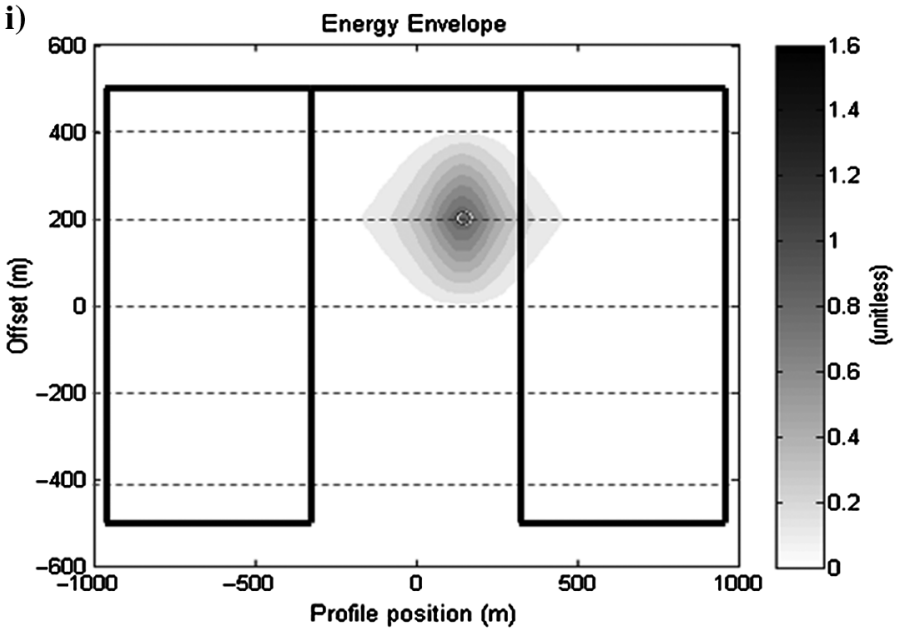

Figure 4. The (a) $x$-component (b) $y$-component, and (c) $z$-component response transverse views; (d) $H_{T}$ (e) $\tilde{H}_{T}$ and (f) EE transverse views; and (g) $H_{T}$ (h) $\tilde{H}_{T}$, and (i) EE plan views for the case in which the magnetic dipole moment is parallel to $[0,1,0]$. The white dot denotes the position at which the fields are a maximum. The gray dot denotes the position of the body. The black lines denote the position of the loops, and the gray dashed lines show the position of the traverse lines. The traverse line running along the center of the configuration is used to plot the transverse views. The peaks of $H_{T}$ and EE coincide with the position of the body, but the peak of $\tilde{H}_{T}$ does not. In this case, $H_{T}$ is the sharpest of all quantities. 
a)

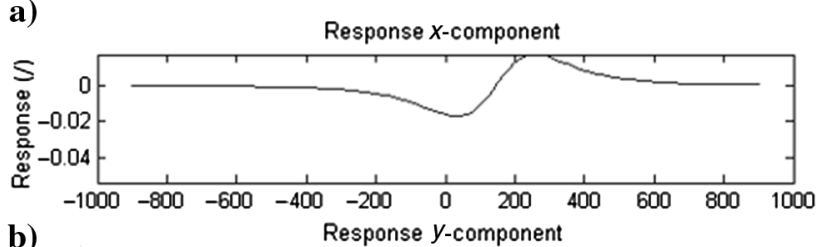

b)

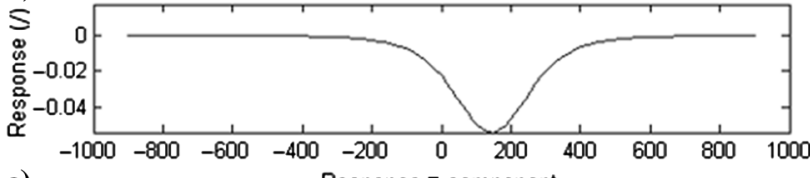

c)

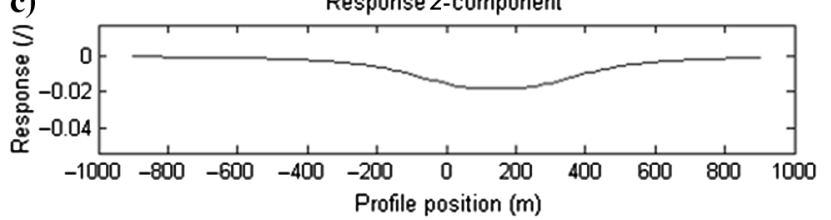

d)

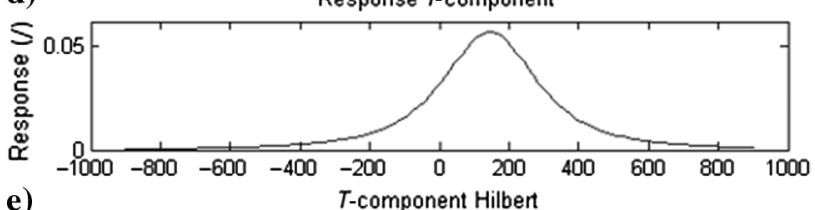

e)

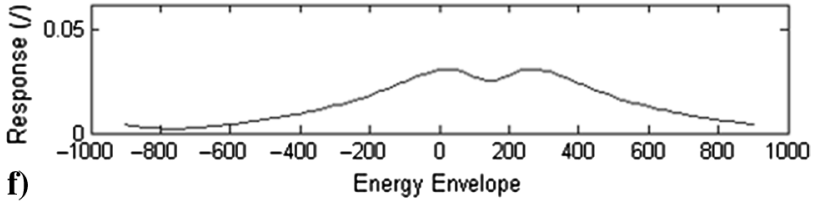

f)

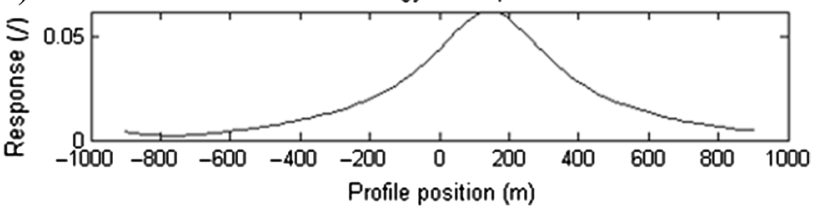

g)

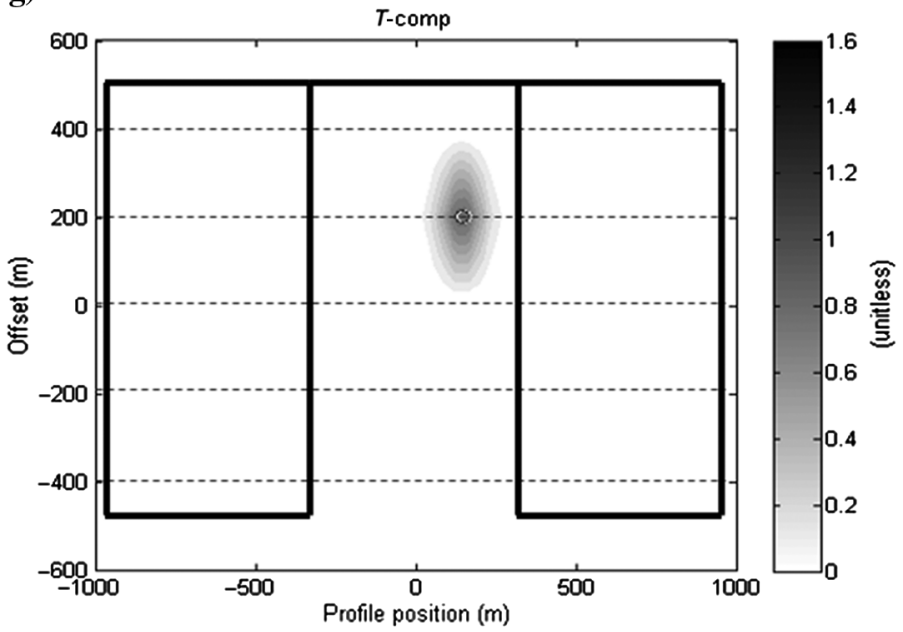

h)

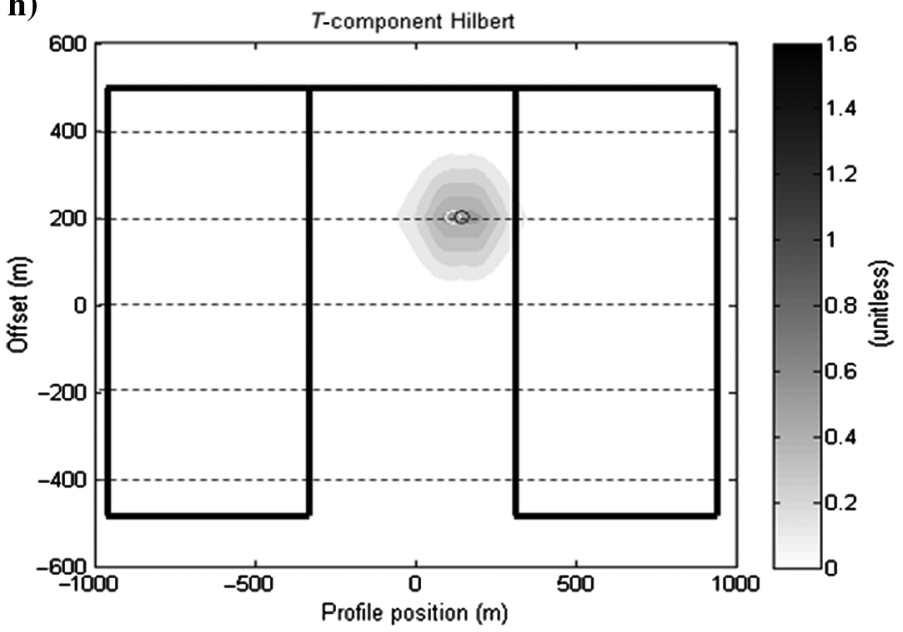

i)

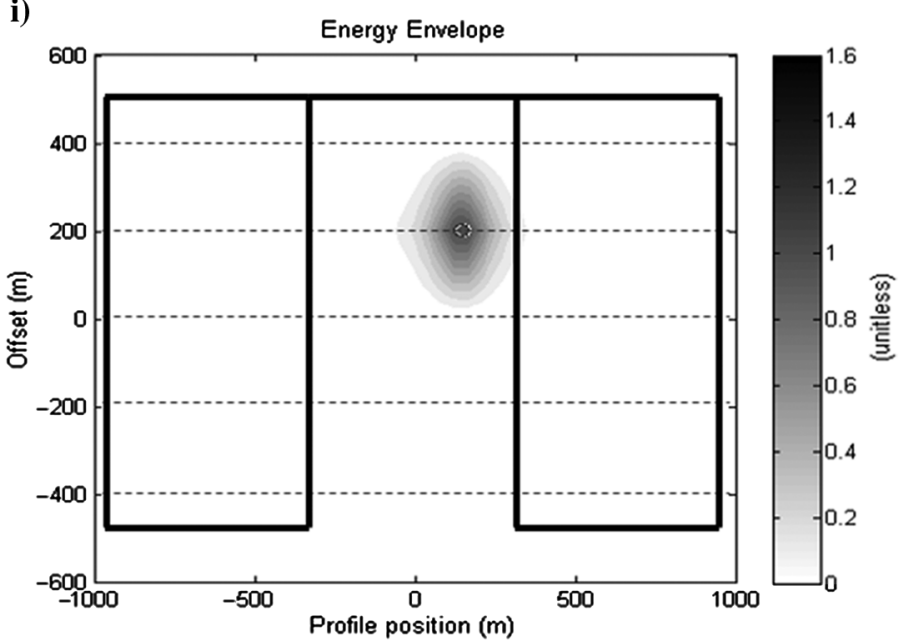

Figure 5. The (a) $x$-component (b) $y$-component, and (c) $z$-component response transverse views; (d) $H_{T}$, (e) $\tilde{H}_{T}$, and (f) EE transverse views; and (g) $H_{T}$, (h) $H_{T}$, and (i) $E E$ plan views for the case in which the magnetic dipole moment is parallel to $[0,0,1]$. The white dot denotes the position at which the fields are a maximum. The gray dot denotes the position of the body. The black lines denote the position of the loops, and the gray dashed lines show the position of the traverse lines. The traverse line running along the center of the configuration is used to plot the transverse views. The peaks of $H_{T}$ and EE coincide with the position of the body, but the peak of $H_{T}$ does not. In this case $H_{T}$ is the sharpest of all quantities. 
shape for small changes in the strike (Desmarais and Smith, 2015b). This change in shape can change the functional dependence on depth and dip for the individual components (Desmarais and Smith, $2015 b$ ). Hence, it is difficult to establish a robust strategy for determining depth and dip from the $x$-, $y$-, and $z$-components. The $T$ component and $T$-component Hilbert-transform response profiles show less variability as a function of strike, so we are advocating that the dip of the target can be approximated from the ratio of the full-width at half-magnitude (FWHM) of the $T$-component response and the $T$-component Hilbert transform response. The depth is subsequently estimated using the FWHM of the $T$-component response.

Figure 6 is a plot of the ratio of the FWHM of the $T$-component response to the FWHM of the $T$-component Hilbert transform response as a function of dip. This plot is generated from a simulation in which the body has a position of [144-, 200-m, depth] with respect to the center of the configuration and a strike of $90^{\circ}$. The station spacing is $15 \mathrm{~m}$. The body is centered directly below the same traverse line as the targets in Figures 3-5. The FWHM was estimated on the $y=200 \mathrm{~m}$ traverse. The light dots are plotted for large depths, whereas the dark dots are plotted for small depths. Depths less than $100 \mathrm{~m}$ have been omitted because the near-surficial primary fields are not reliable. As can been seen from Figure 6, the plotted quantity does not vary significantly as a function of depth, so this plot can be used to approximate the dip.

Figure 7 is a plot of the FWHM of the $T$-component response as a function of depth for the same model as Figure 6. The variation of FWHM magnitude for a dip of $<60$ is negligible, so only one representative line is included. The dip is estimated from Figure 6, so the depth can be estimated as the depth at which the relevant dip curve crossed the estimated FWHM value. The slope and relative position of the curves in Figures 6 and 7 will change according to the orientation of the primary field at the target. Consequently, Figures 6 and 7 depend on the position of the conductor along and transverse to the traverse line and the geometry of the transmitter loops. Because the simulated target is located between the loops and

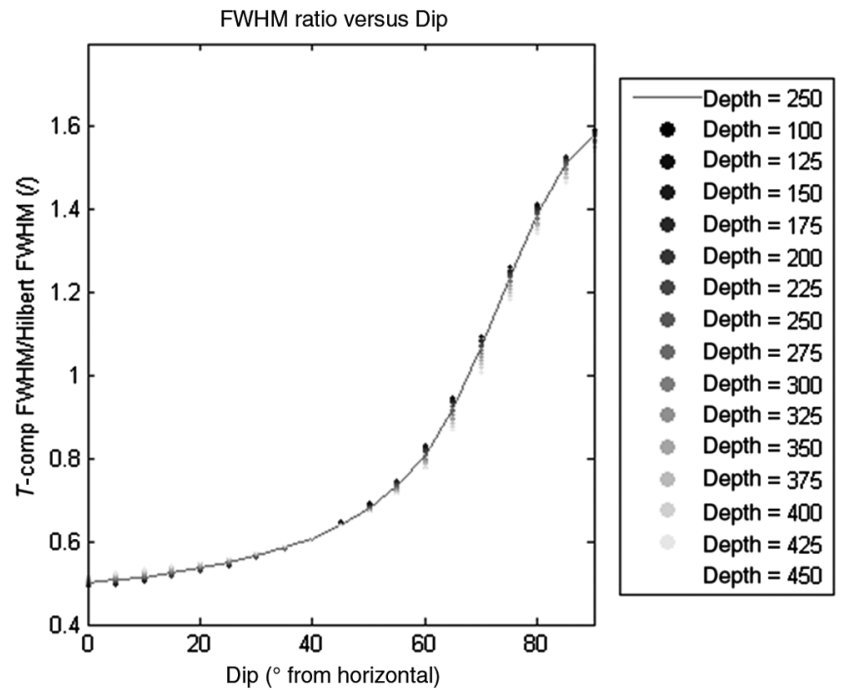

Figure 6. FWHM ratio of the $T$-component response and the $T$-component Hilbert transform response as a function of dip. The light dots are plotted for large depths, whereas the dark dots are plotted for small depths. the fields between the loops are mostly uniform and horizontal (Desmarais and Smith, 2015a), Figures 6 and 7 can be used to obtain good results when investigating targets located in-between the loops and striking perpendicular to the traverse line when using a similar loop geometry. For better results, or when applied to a target with different strike and locations as well as different transmitter loop geometries, the approach outlined above can be used to determine depth and dip, by generating similar plots associated with the pertinent survey parameters.

Quantifying the effects of the variable-loop geometry and target position/orientation on the calculated FWHM and FWHM-ratio parameters is beyond the scope of this study. The slope and relative position of the curves will change according to the target and survey parameters. However, the optimal survey and target parameters of an InfiniTEM system are determined by Desmarais and Smith (2015a), and these are the parameters that were used to generate Figures 6 and 7, so these figures should be applicable to most future InfiniTEM surveys.

The effect of noise should not appreciably change the predicted parameters because an accurate FWHM can still be obtained from a noisy signal. The approach adopted in this paper assumes that the size of the conductor is small compared with the distance between the conductor and the transmitter-receiver system, so it may be approximated as a dipole. This method may not be applicable to conductors of a size comparable with its depth. The method also assumes that the target is in free space, so it may not apply to situations in which the conductor is thick and the background is conductive, so appreciable galvanic interactions occur between the conductor and the background. We believe that our method provides an alternative to EM inversions. If the above approximations are met (no galvanic interactions, conductor small compared with its depth), the method is free from empirical parameterizations, in contrast to the former.

The proposed approach could be used by generating plots for both $B$ field measurements or $\partial B / \partial t$ measurements. Indeed, within the dipole approximation, the response shape is not a function of time (Desmarais and Smith, 2015b). As such, the response can be modeled at any arbitrary delay time. However, in practice, it

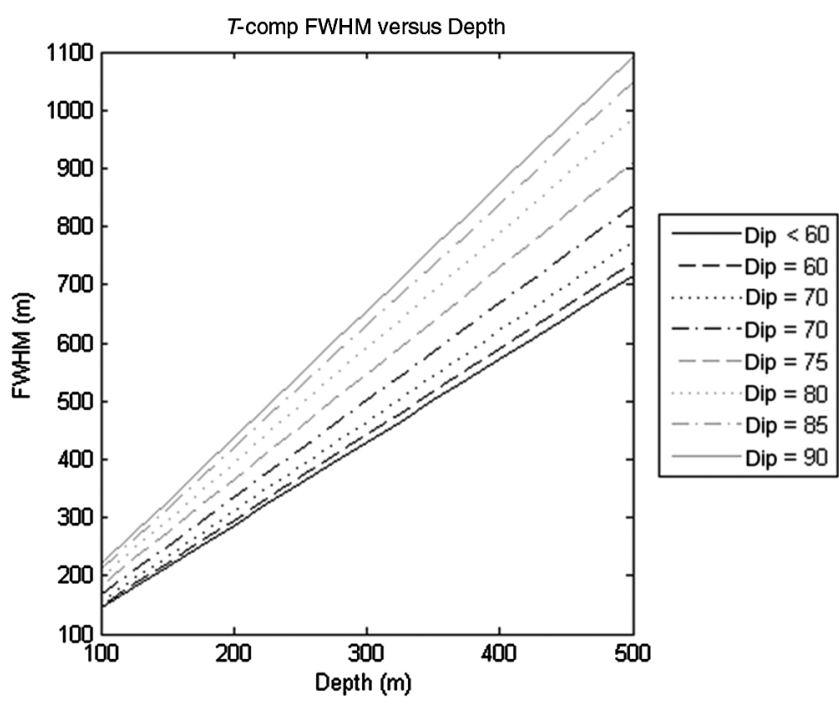

Figure 7. FWHM of the $T$-component response as a function of depth. The variation of FWHM magnitude for a dip of $<60$ is negligible, so only one representative line is included. 
is recommended that the delay time yielding the largest signal-tonoise ratio be used for modeling. As discussed by Desmarais and Smith (2015a), $B$-field measurements are most sensitive to highly conductive targets, whereas $\partial B / \partial t$ measurements are most sensitive
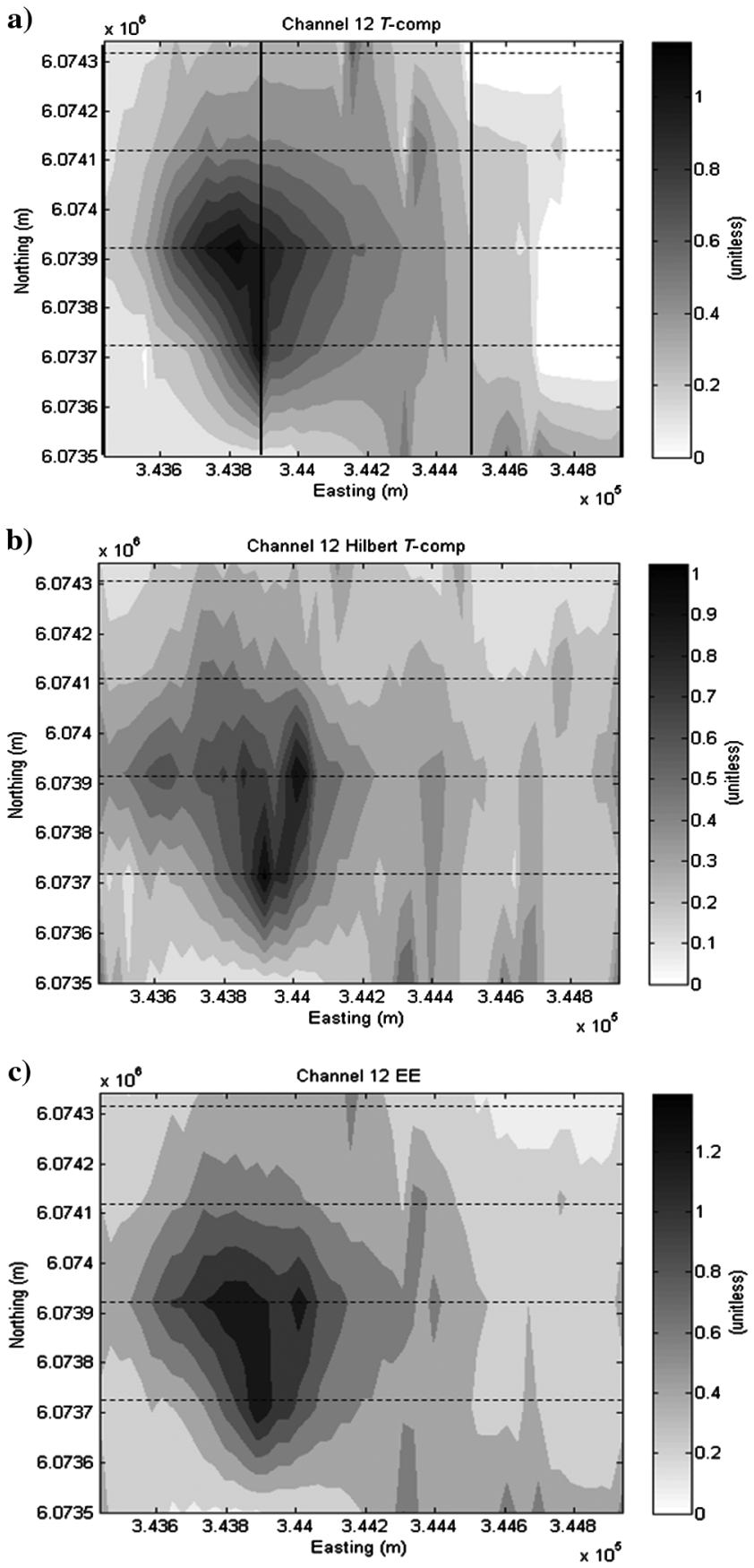

Figure 8. The (a) $T$-component response, (b) $T$-component Hilberttransform response, and (c) EE acquired over the northernmost anomaly at the Coulon field site. The dotted lines denote approximate traverse-line positions. The black lines in panel (a) denote the approximate loop positions. The loops extend $\sim 200 \mathrm{~m}$ north and south of the figure. The EE is sharper than the $T$-component suggesting that the strike and dip are high. The EE and $T$-component peaks are elongated normal to the traverse line suggesting that the strike is $\sim 90^{\circ}$. to less conductive targets. The range of conductivity over which $B$ field or $\partial B / \partial t$ measurements are most suitable depends on the shape of the transmitter current waveform. In practice, it is proposed that the $B$ field and $\partial B / \partial t$ measurements are plotted as a $T$-component response and that the measurements yielding the greatest signal-tonoise ratio be used to extract the geometric parameters of the target.

\section{Tests over the Coulon field site}

Our approach for modeling ground TDEM data was tested over the Coulon field site in the Abitibi greenstone belt, Quebec. For this particular survey, the loop widths were $500 \mathrm{~m}$ along the east direction and $1200 \mathrm{~m}$ along the north direction. The loops were separated by $600 \mathrm{~m}$, and the traverse line spacing was $200 \mathrm{~m}$. The loop geometry is different from that used in the synthetic models because these data were acquired prior to the publication of the Desmarais and Smith (2015a) study, so the optimal loop geometry was not yet known. The station spacing was $30 \mathrm{~m}$, and the data were acquired in 20 channels using a waveform base frequency of $30 \mathrm{~Hz}$ and a duty cycle of $50 \%$. For a general description of the geologic characteristics of the field area, we refer readers to the paper of Thurston et al. (2008).

The data were inspected at each channel to determine the delay time yielding the greatest signal-to-noise ratio. For this particular anomaly, it was found that channel $12(1.0800 \mathrm{~ms})$ yielded the greatest signal-to-noise ratio. The data were subsequently normalized to the largest value of the $x$-, $y$-, and $z$-components over the entire survey area. Finally, the data and derived data were plotted in plan format.

Figure 8 shows the three-component TDEM response acquired over the anomaly, along with its associated $T$-component response and EE. Upon inspecting Figure $8 \mathrm{a}$ and $8 \mathrm{c}$, it is evident that the peaks of the $T$-component response and the EE are elongated normal to the traverse line, suggesting a strike of $\sim 90^{\circ}$. The fact that the EE appears sharper than the $T$-component response suggests that the magnetic dipole moment of the target is nearly parallel to the $x$-direction (the strike is $\sim 90^{\circ}$, and the dip is close to vertical). The $T$-component response FWHM is $889 \mathrm{~m}$, and the $T$-component to $T$-component Hilbert-transform response FWHM ratio is 0.95 . The dip and depth of the target can be determined from these parameters upon consulting Figure 9a and 9b, which are plots of the FWHM ratio and the $T$-component response FWHM for the appropriate survey and target parameters. Figure 9 is different from Figures 6 and 7 because of the difference in the loop geometry. Figure 9 yields a dip of $70^{\circ}$ and a depth of $475 \mathrm{~m}$ for the associated target.

Figure 10a is a geologic map of the area associated with the anomaly of Figure 9. The coordinates of the location are identical to those of Figure 9, and the $T$-component response is included alongside in Figure 10b for comparison. As can be seen from Figure 10, the highlighted interface between the two lithologies coincides approximately with the asymmetry of the $T$-component response and is oriented at a strike $\sim 90^{\circ}$. Indeed, the asymmetric peak of the $T$-component response does not coincide perfectly with the highlighted interface. This is because the position of the lithologies and of the EM fields are approximate and because a plan view of the lithological contact most likely varies as a function of depth, so its surface projection may not fully reflect the position of the conductor. As well, the peak of the $T$-component response is at an angle to the lithological contact. This occurs because the EM fields have been sampled at a coarse line spacing, so the strike 
of the $T$-component peak is not fully resolved. However, it should be clear from Figure 10 that the $T$-component peak follows the general trend of the lithological contact.

a)
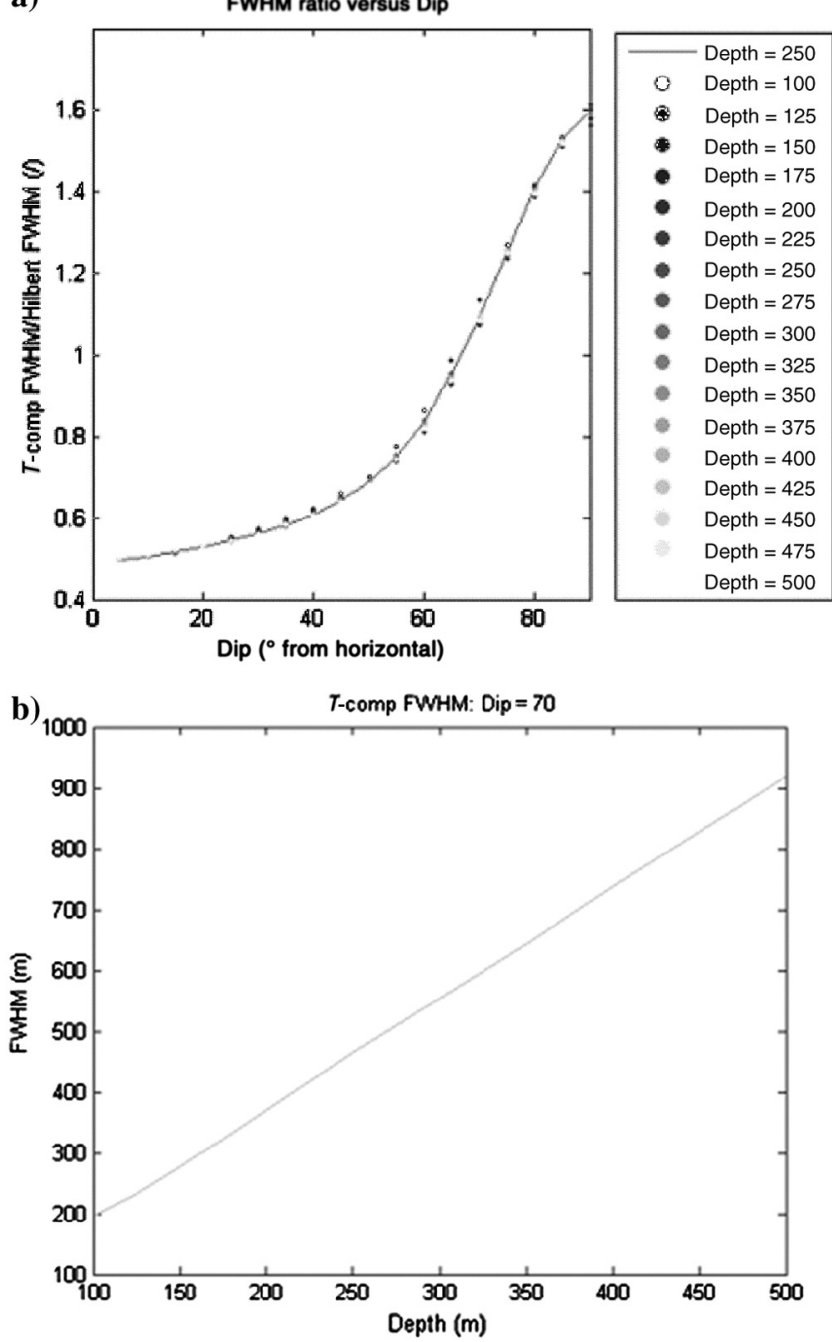

Figure 9. (a) FWHM ratio and (b) $T$-compoent FWHM for a simulation associated with the northernmost anomaly at the Coulon field site. The dip is approximated at $70^{\circ}$ using panel (a), and the depth is approximated at $475 \mathrm{~m}$ using panel (b).
Considering that the field area is located in the Abitibi greenstone-belt (an Archean volcanic terrane), two geologic models may be responsible for the observed anomaly. The anomaly may either be caused by a fault or a volcanogenic-massive sulfide (VMS) lens. Faulting typically occurs along geologic anisotropies, and VMS lenses are generally stratiform. In both cases, a strike of $90^{\circ}$ should be expected. As well, in Archean terranes diapirical plutonism has rendered volcanic strata vertical, and this is consistent with the high value of the dip obtained from our modeling results (Van Kranendonk et al., 2004; Thurston et al., 2008). The fact that lithological interfaces are visible on a plan map suggests that volcaniclastic bedding has indeed been overturned. Our modeling results are therefore consistent with the current outstanding geologic model of the field area.

\section{CONCLUSION}

The geometric parameters of a relatively small conductor can be estimated using combinations of spatial components and their Hilbert transforms, as acquired from three-component ground-TDEM data. The position of the target can be determined from the location of the peaks of the EE or the $T$-component response. The EE is sharper than the $T$-component response when the magnetic dipole moment of the target is oriented parallel to the $[1,0,0]$ direction. Whereas the $T$-component response is sharper than the EE when the magnetic dipole moment of the target is oriented parallel to the $[0,1,0]$ and $[0,0,1]$ directions. Hence, the $T$-component response is most suitable for data interpretation in most possible target orientations. Once the position of the body has been determined, the dip of the target can be quantified using the ratio of the FWHM of the $T$-component response and the $T$-component Hilbert transform response. Finally, once the dip been determined, the depth of the target can be evaluated using the FWHM of the $T$-component response.

The proposed modeling method was tested over an anomaly acquired from an InfiniTEM survey at the Coulon field site in the Abitibi greenstone belt of Quebec. Our method predicts that the conductor responsible for the anomaly has a strike of $90^{\circ}$, a dip of $70^{\circ}$, a position of $6.0740 \mathrm{E} 6 \mathrm{~m}$ northing, 3.438E5 $\mathrm{m}$ easting, and a depth of $475 \mathrm{~m}$. These parameters are consistent with an interpretation based on the current outstanding models of the Archean greenstone belt formation. a)

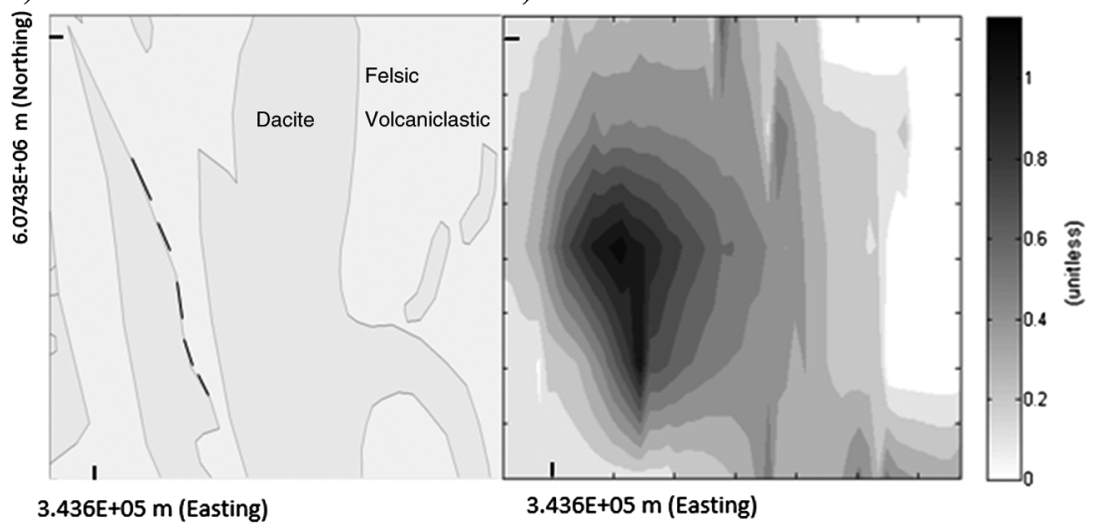

Figure 10. (a) Lithological map of the area associated with the anomaly of Figure 8. Rock types are annotated, and the coordinates of the location are similar to those of Figure 8. (b) The $T$-component response is included for comparison. The highlighted interface between the dacite and the felsic volcaniclastics coincides with the peak of the $T$-component response. The distance between tick marks on the maps is $200 \mathrm{~m}$. 


\section{ACKNOWLEDGMENTS}

We are grateful to Abitibi Geophysics and Virginia Mines for directing us to appropriate geologic information. In particular, we thank M. Savard of Virginia Mines and P. Bérubé, R. Wasylechko, and J. Collins of Abitibi Geophysics. We also thank E. Gasperikova, J. Shragge, as well as three anonymous reviewers whose suggestions have helped improve this paper.

\section{REFERENCES}

Akgün, M., 2001, Estimation of some bodies parameters from the self potential method using Hilbert transform: Journal of the Balkan Geophysical Society, 4, no. 2, 29-44.

Bérubé, P., M. Boivin, C. Malo-Lalande, and P. Paré, 2006, Transmitter loops in series for electromagnetic geophysical surveys: U.S. Patent $7,116,107 \mathrm{~B} 2$.

Bournas, N., and H. A. Baker, 2001, Interpretation of magnetic anomalies using the horizontal gradient of the analytical signal: Annali di Geofisica, 44, 505-526, doi: $10.4401 \% 2$ Fag-3572.

Brakni, M., 2011, Réseaux de neurones artificiels appliqués à la méthode électromagnétique transitoire InfiniTEM: M.S. thesis, Université du Québec en Abitibi-Témiscamingue.

Cox, L. H., G. A. Wilson, and M. S. Zhdanov, 2010, 3D inversion of airborne electromagnetic data using a moving footprint: Exploration Geophysics, 41, 250-259, doi: 10.1071/EG10003.

Debeglia, N., and J. Corpel, 1997, Automatic 3-D interpretation of potential field data using analytic signal derivatives: Geophysics, 62, 87-96, doi: 10 $.1190 / 1.1444149$.

Desmarais, J. K., and R. S. Smith, 2015a, Survey design to maximize the volume of influence of the InfiniTEM system, when looking for discrete targets: Journal of Applied Geophysics, 115, 11-23, doi: 10.1016/j .jappgeo.2015.02.012.

Desmarais, J. K., and R. S. Smith, 2015b, Decomposing the electromagnetic response of magnetic dipoles to determine the geometrical parameters of a dipole conductor: Exploration Geophysics, doi: 10.1071/EG14070.
Macnae, J. C., 1984, Survey design for multicomponent electromagnetic systems: Geophysics, 49, 265-273, doi: 10.1190/1.1441658.

Malo-Lalande, C., 2007, An InfiniTEM survey leads to a new discovery on the Coulon property: Proceedings of Exploration 07: Fifth Decennial International Conference on Mineral Exploration, 1107-1110.

Mercer, A., 2012, Combining multiple components of InfiniTEM electromagnetic data to better represent subsurface anomalies: M.S. thesis, Memorial University of Newfoundland.

Nabighian, M. N., 1974, Additional comments on the analytic signal of twodimensional magnetic bodies with polygonal cross-section: Geophysics, 39, 85-92, doi: 10.1190/1.1440416.

Nabighian, M. N., 1984, Toward the three-dimensional automatic interpretation of potential field data via generalized Hilbert transforms: Fundamental relations: Geophysics, 49, 780-786, doi: 10.1190/1.1441706.

Nabighian, M. N., and J. C. Macnae, 1972, The analytical signal of twodimensional magnetic bodies with polygonal cross-section: Its properties and use for automated anomaly interpretation: Geophysics, 37, 507-517, doi: 10.1190/1.1440276.

Oppenheim, A. V., R. W. Schafer, and J. R. Buck, 1998, Discrete-time signal processing: Prentice Hall.

Smith, R. S., and P. B. Keating, 1996, The usefulness of multicomponent, time-domain airborne electromagnetic measurements: Geophysics, 61, 74-81, doi: 10.1190/1.1443958.

Smith, R. S., and T. J. Lee, 2001, The impulse-response moments of a conductive sphere in a uniform field, a versatile and efficient electromagnetic model: Exploration Geophysics, 32, 113-118, doi: 10.1071/EG01113.

Thurston, P. C., J. A. Ayer, J. Goutier, and M. A. Hamilton, 2008, Depositional gaps in the Abitibi Greenstone Belt stratigraphy: A key to exploration for syngenetic mineralization: Economic Geology, 103, 10971134, doi: 10.2113/gsecongeo.103.6.1097.

Van Kranendonk, M. J., W. J. Collins, A. Hickman, and M. J. Pawley, 2004, Critical tests of vertical vs. horizontal tectonic models for the Archean East Pilbara granite-greenstone terrane, Pilbara Craton, Western Australia: Precambrian Research, 131, 173-211, doi: 10.1016/j.precamres.2003.12 .015 .

Yang, D., D. W. Oldenburg, and E. Haber, 2014, 3-D inversion of airborne electromagnetic data parallelized and accelerated by local mesh and adaptive soundings: Geophysical Journal International, 196, 1942-1507, doi: $10.1093 /$ gji/ggt465. 